\title{
Thymic Carcinoid: A Rare Case Presentation
}

\author{
Ashfaq UI Hassan \\ Anatomy SKIMS Medical College Bemina \\ *Corresponding Author: ashhassan@rediffmail.com
}

Copyright (C) 2013 Horizon Research Publishing All rights reserved.

\begin{abstract}
Carcinoid tumors are not a common entity in thorax. The most common sites include the abdominal sites like the Appendix and the Ileum. The carcinoids in the thorax are seen rarely and that to incidentally.in thymus the carcinoids are all together rare. Usually carcinoids tend to be Chromaffin positive histologically and secrete a largr number of chemicals. They belong to the APUD system(Amine Precursor Uptake and decarboxylation system). Thymic carcinoid is a distinct entity which is very rare. It is a slow growing but potentially malignant tumor. We present a rare case of thymic carcinoid in a 16 year old male. Fortunately the disease was confined to Thymus and there was no metastatic spread to other organs. Cytological examination of samples obtained by ultrasound guided fine needle aspiration provided the diagnosis in the patient.
\end{abstract}

Keywords Thymus, Neuroendocrine, Metastasis, Chromogranin, Synaptophysin

\section{Introduction}

Thymic carcinoid is a distinct entity which is very rare. It is a slow growing but potentially malignant tumor.

We present a rare case of thymic carcinoid in a 16 year old male. Fortunately the disease was confined to Thymus and there was no metastatic spread to other organs. Cytological examination of samples obtained by ultrasound guided fine needle aspiration provided the diagnosis in the patient.

\section{Case Report}

A 16 years old male presented with gradual onset chest pain and dyspnoea of two months .Examination revealed a $48 \mathrm{~kg}$ weight male with no obivious lymphadenopathy. CT scan of the thorax revealed a large well defined heterogenous density anterior mediastinal mass. Abdominal ultrasonography did not revealed any metastatic lesion or organomegaly. Routine blood investigations were within normal limits. The tumor had synaptophysin positivity, chromogranin a positivity.

\section{Discussion}

Thymic carcinoids are the Neuroendocrine tumours of Thymus ${ }^{1}$ being more common in males ${ }^{2}$. They Belong to the APUD System Of cells (The amine precursor uptake and decarboxylation (APUD) system represents cells that are endocrine in function and produce hormones, neuropeptides, and paracrine agents. They have high amine content in their cytoplasm, are capable of amine precursor uptake, and are able to decarboxylate these to produce amines and/or peptides.

As compared to other tumors they are very rare.

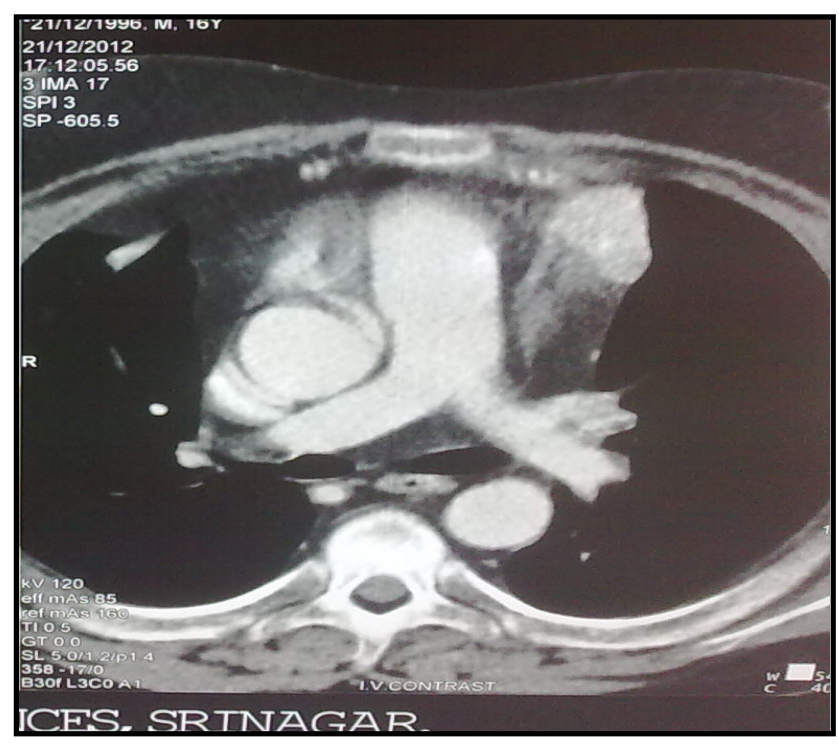

Figure 1. CT Scan demonstrating Anterior Mediastinal mass in the form of Thymic tumor

Histologically, the thymic neuroendocrine carcinomas have been divided in three categories: Low-grade (well-differentiated), intermediate-grade (moderately differentiated) and high-grade (poorly differentiated) tumors.

The tumour cells contain neurosecretory granules which contain a wide variety of peptides and amines, such as 5-hydroxytryptamine (5-HT), neuron-specific enolase, hydroxytryptophan, synaptophysin, chromogranin A and $\mathrm{C}$ and several peptides such as insulin, growth hormone, adrenocorticotrophic hormone (ACTH) and gastrin. ${ }^{4,5,6}$ 
The immune-histochemical stains usually provide useful characteristic patterns for thymic neuroendocrine tumors. Markers such as chromogranin, Leu-7, neuron specific enolase, bombesin, and synaptophysin can also help to identify these lesions. Sometimes other markers may also be present in these tumors-melanocyte, serotonin, somatostatin, cholecystokinin, neurotensin, metenkephalin and S-100 protein.

Radiologically, these tumours typically manifest as large masses with a propensity for local invasion. Focal areas of necrosis and punctate calcification may be present ${ }^{7}$

Mediastinal carcinoid tumors arise from cells of Kulchitsky located mostly in the thymus. Occurring more often in male patients, these tumors are usually located in the anterosuperior mediastinum. Owing to their origin from APUD cells, these tumors may be hormonally active, and they may occur as a variant of the multiple endocrine neoplasia syndromes.

Thymic carcinoids can be frequently associated with Cushing's syndrome due to production of ACTH and less frequently with multiple endocrine neoplasia Type 1 syndrome.

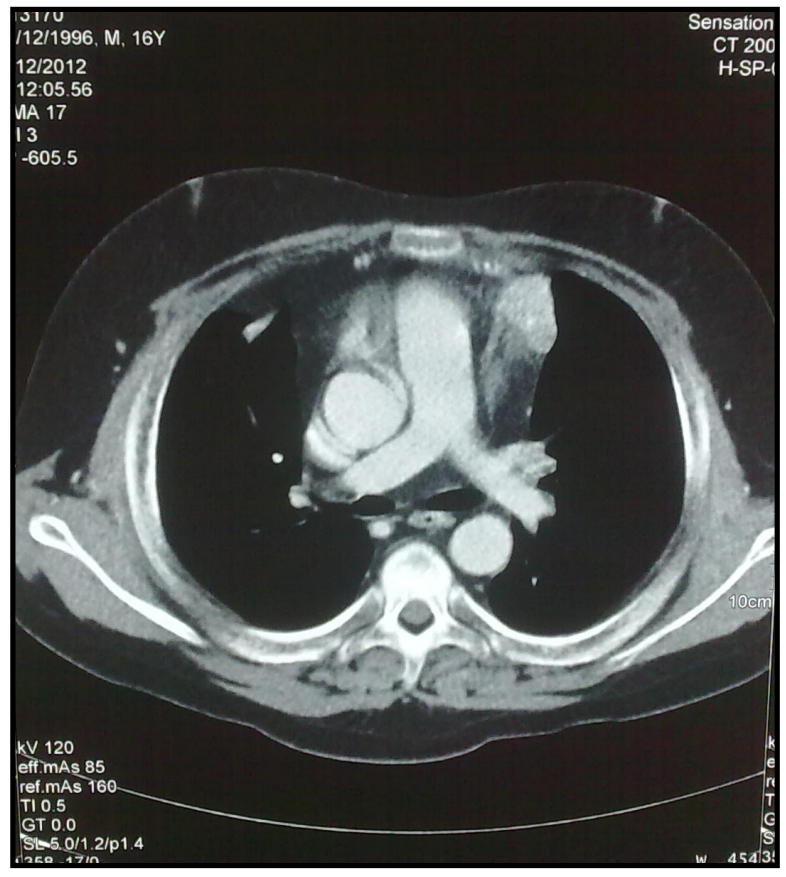

Figure 2. CT Scan demonstrating Thymic Carcinoid

In patients with hormonally inactive tumors, symptoms are related to local mass effects, leading to chest pain, dyspnea, cough, and the superior vena caval syndrome. Hormonally inactive carcinoids tend to be larger and frequently are invasive locally. In addition, metastatic spread to mediastinal and cervical lymph nodes, liver, bone, skin, and lungs occurs in the majority of patients.

Often, these tumors are difficult to differentiate from other common anterior mediastinal masses, particularly thymomas and germ cell tumors.

However, carcinoids are characterized by the ultrastructural findings of dense core neurosecretory granules. Positive immunohistochemical staining of these granules for ACTH is also characteristic.

Surgical removal of the tumor, when possible, is the preferred treatment. When local invasiveness or metastasis precludes the successful use of operative therapy, radiation therapy and multiagent chemotherapy have been used, although no consistent benefit has been documented.

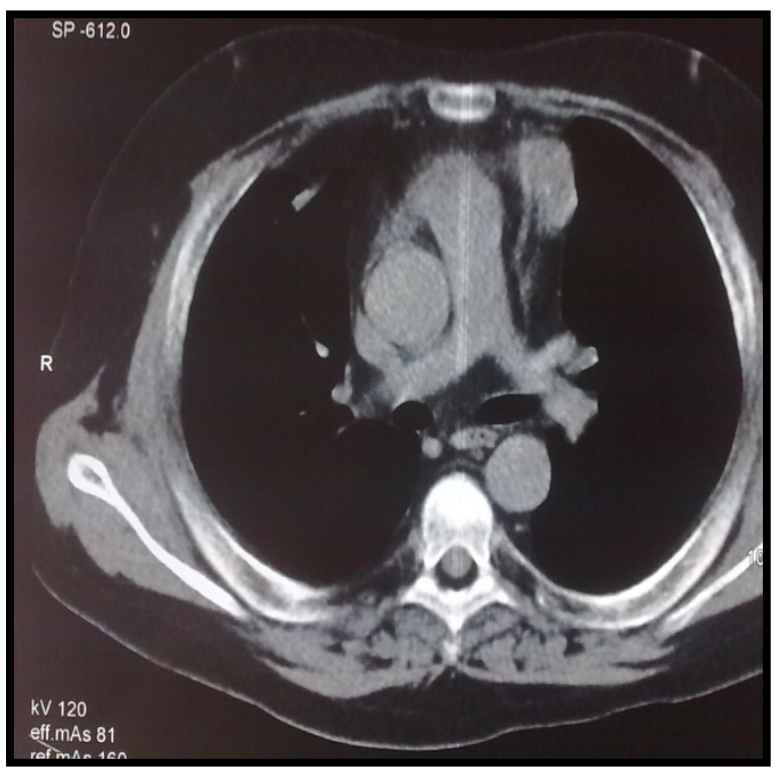

Figure 3. CT Scan demonstrating Anterior Mediastinal mass(Carcinoid in Thymus)

\section{Conclusion}

The primary neuroendocrine tumors of the thymus are very aggressive tumors which are associated with poor survival. Surgical resection of the tumor and its extension remains the treatment of choice because the experience with adjuvant therapy with 5-flurouracil, streptozocin, carmustine, VP-16, Cisplatin has been unsatisfactory. Thymic neuroendocrine carcinomas have a rather poor prognosis based on their tendency to recur and metastasize many years after the initial operation. Therefore, prolonged follow-up is essential for these tumors.

\section{REFERENCES}

[1] Brant WE, Helms CA. Fundamentals of diagnostic radiology. Lippincott Williams \& Wilkins. (2007)

[2] Nishino M, Ashiku SK, Kocher ON et-al. The thymus: a comprehensive review. Radiographics. 26 (2): 335-48. doi:10.1148/rg.262045213

[3] Rosado De Christenson ML, Abbott GF, Kirejczyk WM et-al. Thoracic carcinoids: radiologic-pathologic correlation. Radiographics. 1999;19 (3): 707-36. Radiographics (full text) 
- Pubmed citation

[4] Jensen RT, Doherty GM. Carcinoid tumors and the carcinoid syndrome. In: Devita VT, Hellman S, Rosenberg SA, editors. Cancer. Principles and Practice of Oncology. Philadelphia: Lippincott/Raven; 2001. pp. 1813-33.

[5] Capella C, Heitz PU, Hofler H, et al. Revised classification of neuroendocrine tumours of the lung, pancreas and gut. Virchows Arch. 1995;425:547-60. [PubMed]

[6] Pantongrag-Brown L, Buetow PC, Carr NJ, et al. Calcification and fibrosis in mesenteric carcinoid tumor: CT findings and pathologic correlation. Am J Roentgenol. 1995;164:387-91. [PubMed]

[7] Brown LR, Aughenbaugh GL. Masses of the anterior mediastinum: CT and MR imaging. AJR Am J Roentgenol. 1991; 157(6): 1171-80. AJR Am J Roentgenol 